\title{
Discussion on IPRAN Transmission Network Development for 5G Evolution
}

\author{
Libo Chen \\ China Information Technology Designing \& Consulting Institute CO.LTD Chengdu Branch, Chengdu, \\ Sichuan, 610042 \\ chenlb@dimpt.com
}

Keywords: IPRAN, Grouping, Internet of Things, 5G Evolution

\begin{abstract}
In order to analyze the mobile communication in the next few years in the evolution from $4 G$ to $5 G$ on transmission especially IPRAN network, this paper is based on the changes of characteristic of the wireless network and capacity, connection and time delay sensitivity features such as a sharp rise. It analyzes the development demand for transmission network, based on the 5G demand, puts forward target transmission network architecture, carries a plan of prequel and postpass, and calculates the bandwidth within the metropolitan area transmission network, discusses the metro IPRAN for 5G network evolution direction of the core layer, convergence layer and accesess layer and technical characteristics.
\end{abstract}

\section{Introduction}

A new generation of mobile communications technology every decade, the current 4G has entered a large-scale commercial stage. As the next trillion-scale strategic emerging industries, 5G has become the focus of international competition.

$5 G$ network will provide ultra-high access speed, ultra-low latency, ultra-reliable user experience to support a variety of mobile Internet and Internet of Things business needs to meet the ultra-high traffic density, high connection density and high mobility of the access requirements, while the network will bring ultra-fold increase in energy efficiency and ultra-hundred times the bit cost reduction, and several times the spectral efficiency.

Through the opening of network capabilities, 5G provides flexible business deployment environments to third parties for friendly interaction with third-party applications. NFV and SDN technology to promote the innovation of 5G network architecture, network functions can be arranged on demand, operators can flexibly combine functional modules according to different scenarios and business characteristics, customize network resources and business logic as needed, and enhance the network Flexible and adaptive.

\section{5G network hosting needs}

With the rapid development of mobile Internet and Internet of Things market and business applications, 5G technology has become the focus of the industry in recent years, and research on $5 \mathrm{G}$ bearer solutions has also been put on the agenda.

The 5G technology targets the mobile Internet and the Internet of Things (IoT), and mainly covers three technical scenarios: Enhanced Mobile Broadband (eMBB) (Continuous Wide Area Coverage, Hot Spot High Capacity), Large Machine Communication (mMTC) And high reliability low latency communication scenario (uRLLC). The first scenario is mainly for mobile Internet applications, the latter two scenarios mainly for the Internet of Things and the vertical industry applications.

As shown in Table 1, by analyzing the main application scenarios of 5G, the demand of the bearer network of the $5 \mathrm{G}$ wireless network can be refined:

1) bandwidth requirements. Continuous wide area coverage scenario is the most basic coverage of mobile communications, users need to provide more than 100Mbps user experience anytime, anywhere; hot high-capacity scene for local hot spots, you need to meet the user's high data transfer 
rate and the regional range of the very High data traffic requirements include 1Gbps user experience rate, $10 \mathrm{Gbps}$ peak rate and $10 \mathrm{Tbps} / \mathrm{km} 2$ traffic density;

2) low latency, high reliability requirements. Vertical industries such as car networking and industrial control are demanding for latency and reliability, with millisecond-level end-to-end latency and almost 100\% reliability. This requires bearer network can provide very low transmission delay, low processing delay, strict frequency synchronization and time synchronization capabilities, but also provide a strong fault recovery capability.

3) Low TCO demand. Wide area coverage, high density, large capacity and large bandwidth of 5G networks will inevitably have heavy demand on the bearer network elements and the scale of bearer networks will be huge. This requires that the bearer network elements have low cost, low power consumption, easy maintenance, and maximum reduction TCO.

4) Intelligent needs. 5G network takes SDN as the basic technology, the control plane and the forwarding plane are separated, and the entire network is more flexible, intelligent, efficient and open. As part of the 5G forwarding plane, the bearer network must also have the SDN function to build a service-oriented network capability open interface that supports multi-domain coordination and network fragmentation to meet the differentiation needs of the service and improve the deployment efficiency of the service.

5) equipment, large-capacity, large bandwidth, the number of interfaces and more demand. In the era of 5G, the number of bearer network equipment is large. There are many scenarios of MESH topologies, such as large bandwidth and interface consumption, which require large capacity, large bandwidth, and large number of interfaces.

Table 1 5G application scenarios features and requirements

\begin{tabular}{|c|c|c|}
\hline Scenes & Features & Indicator requirements \\
\hline $\begin{array}{l}\text { Enhanced } \\
\text { Mobile } \\
\text { Broadband } \\
\text { (eMBB) }\end{array}$ & $\begin{array}{l}\text { Under certain scenarios to } \\
\text { provide users with high data } \\
\text { transfer rate, } 1 \mathrm{Gbps} \text { user } \\
\text { experience rate, dozens of } \\
\text { GPS peak rate (scene: indoor } \\
\text { ultra-high-bandwidth access) }\end{array}$ & $\begin{array}{l}\text { - } \quad \square \text { User experience rate, downlink 1Gbps, } \\
\text { uplink 500Mbps } \\
\text { - } \quad \square \text { Delay, } 10 \mathrm{~ms} \\
\text { - } \quad \square \text { Peak rate, } 20 \mathrm{Gbps}\end{array}$ \\
\hline $\begin{array}{l}\text { Large-scale } \\
\text { machine } \\
\text { communication } \\
\text { (mMTC) }\end{array}$ & $\begin{array}{l}\text { Sensor-oriented and data } \\
\text { acquisition applications such } \\
\text { as scenarios require the } \\
\text { network to support } \\
\text { ultra-billion connections, } \\
\text { while ensuring the terminal's } \\
\text { ultra-low power consumption } \\
\text { and cost }\end{array}$ & $\begin{array}{l}\text { ? experience rate, 1-100Kbps } \\
\text { Latency, seconds to hours } \\
\text { ? connection number density, up to } \\
00000 \text { / km2 }\end{array}$ \\
\hline $\begin{array}{l}\text { High reliability } \\
\text { and low latency } \\
\text { communication } \\
\text { (uRLLC) }\end{array}$ & $\begin{array}{l}\text { Car networking and other } \\
\text { applications for the scene, the } \\
\text { transmission delay and } \\
\text { reliability with high } \\
\text { requirements }\end{array}$ & $\begin{array}{l}\text { ? } \text { ? experience rate, downlink 50kbps } \\
0 \mathrm{Mbps} \text {, up a few bps } \sim 10 \mathrm{Mbps} \\
0 \quad \text { ? Delay, } 1 \mathrm{~ms} \\
-\quad \text { ? moving speed, } 0 \bullet 50 \mathrm{~km} / \mathrm{h}\end{array}$ \\
\hline
\end{tabular}

\section{5G bearer network program analysis}

$4 \mathrm{G}$ network is mainly for mobile Internet, is a part of $5 \mathrm{G}$ network and lays a good foundation for the construction of $5 G$ network. However, the requirements of $5 G$ networks are more stringent than those of $4 \mathrm{G}$ networks. Compared with $4 \mathrm{G}$ networks, 5G networks are far more targeted than 4G networks. The evolution to $5 \mathrm{G}$ networks based on $4 \mathrm{G}$ networks can not fully meet the demand of $5 \mathrm{G}$ for Internet of Things. Therefore, in view of protecting historical investment and reducing the construction cost of 5G network, for mobile Internet, operators will choose to evolve to 5G based on 4G network. For Internet of Things, operators will adopt a new strategy. The bearer network is part of the 4G / $5 \mathrm{G}$ network and operators will adopt the same strategy.

In the era of 5G, the network further flattened and the "service cloud" further subsided. The vertical delay of "service cloud" to "service end" and the horizontal delay of "service end" to 
"service end" were greatly reduced. For the bearer network Delay requirements more stringent than the $4 \mathrm{G}$ era, bearer network must be flattened.

The traditional bearer network is divided into access layer, convergence layer, backbone convergence layer, core layer, and service floor. In the era of 5G, the bearer network maintains certain access, aggregation, and the core structure of the three- The degree of simplification, the convergence layer will not be the backbone convergence node secondary convergence, after a convergence of the mouth directly to the core layer double-word, instead of the current ring network, and even in the case of equipment capacity permit, the business Strata will be simplified.

5G target bearer network topology shown in Figure 2, the preamble using optical fiber direct drive, coarse wave points and other technologies; In order to meet the requirements of cross-domain cross-domain cross-site traffic flow scheduling, the convergence needs to be done in a ring, the core layer needs to be connected to the core layer, and the service layer is fully connected with the "service cloud" A number of converged ring business scheduling, the core layer is also ring.

The bandwidth requirements of $5 \mathrm{G}$ for the pre-transmission interface mainly include the requirements of bandwidth and delay. If the $5 G$ is measured according to the traditional CPRI interface in the $4 \mathrm{G}$ era, the bandwidth of the pre-transmission will be greatly improved. Taking the S111 base station with 20MHz bandwidth of 8 antennas as an example, 5G single-sector CPRI interface bandwidth increased about 160 times (10 times the spectral bandwidth increase, the number of antennas to enhance 16 times) to 1.6T; and the delay will drop to below 100us.

DU and CU put different requirements on the transmission network in the transmission architecture. For the east-west traffic, one DU will be connected to only one CU. In the D-RAN architecture, there will be east-west traffic. With C-RAN, there is no east-west traffic between the DU and east-west traffic between the DU's Interaction.

Scenario 1: Both CU and DU are converging nodes.

Scenario 2: The CU is at the sink node and the DU is at the integrated service access point.

Scenario 3: Both CU and DU are in the integrated service access point.

The CU will be a concentration of hundreds of base stations, and the location is more likely to be at a convergence point or core point (small city). Equipment shape is not sure. But will consider pool deployment, or IT equipment to cloud deployment.

One DU will only connect to one CU, and the CU will be backed up; the DU should be the same location as LTE. For high-frequency stations (micro-stations) DU may be put together with the AAU, but high-frequency deployment will not be much. The current BBU can support 30 cells, the future hope to bring more than 60 cells.

At present, the pre-transmission interface of $5 \mathrm{G}$ can be solved in 4 ways, such as bare fiber direct drive, passive wavelength division, OTN solution and packet solution. It is currently recommended to use bare fiber direct drive or passive WDM solution. As far as the network architecture is concerned, with the large-scale deployment of small cells such as Small Cells and the widespread adoption of BBU pooling / cloudization, multi-band carrier aggregation applications, forward-looking and backhaul network boundaries are increasingly blurred. Construct a unified mobile bearer network to reduce CAPEX and OPEX.

The $5 G$ backhaul bearer as shown in Figure 1, the backhaul network allocates different traffic models according to the service types, and mainly solves the circuit requirements of the CU. The above requirements for IP forwarding are all above CU. The requirements of IP routing flexibility are higher than that of LTE. The devices in the IPRAN core aggregation layer should be greatly expanded in capacity and replaced with devices.

\section{5G IPRAN network bearer discussion}

For the evolution of 5G, IPRAN high bandwidth carrying capacity and flexible scheduling capabilities are put forward higher requirements. IPRAN toward the 100G port and slot capacity evolution. In the initial deployment of 5G, especially during the transition of Pre 5G deployment, the $5 \mathrm{G}$ bearer network recommends that the front bearer be deployed using optical direct-drive and coarse wavelength division technologies and the target architecture for on-demand capacity 
expansion on the IPRAN network is to be prepended to meet the $5 \mathrm{G}$ base station network bandwidth requirements, Three-tier functional requirements and synchronization needs.

According to the NGMN bandwidth planning principle, the bandwidth requirement of the base station takes MAX (single-cell peak, average * N); assuming that the operator obtains the spectrum resource of $3.4 \mathrm{G}$ to $3.5 \mathrm{G}$ with the bandwidth of $100 \mathrm{Mhz}$ and the wireless base station adopts the configuration of 3 cells and 64T64R, the spectral efficiency of the network reaches the peak 50bit / hz, mean 10bit / hz. The single-station peak bandwidth $=50 \mathrm{bit} / \mathrm{hz} * 100 \mathrm{Mhz}=5 \mathrm{Gbps}$, single-site mean bandwidth $=3 *(10 \mathrm{bit} / \mathrm{hz} * 100 \mathrm{Mhz})=3 \mathrm{Gbps}$. Suppose the operator obtains the spectrum resource of $28 \mathrm{G}$ and the bandwidth of $800 \mathrm{Mhz}$, and the radio base station adopts 3Cells and 2T2R configurations, the peak spectrum efficiency is $25 \mathrm{bit} / \mathrm{hz}$ and the average spectrum efficiency is 4bit $/ \mathrm{hz}$. The peak bandwidth of single station $=25 \mathrm{bit} / \mathrm{hz} * 800 \mathrm{Mhz}=20 \mathrm{Gbps}$, the average bandwidth of single station $=3 *(4 \mathrm{bit} / \mathrm{hz} * 800 \mathrm{Mhz})=9.6 \mathrm{Gbps}$.

This led to 5G single base station access bandwidth requirements: 5Gbps 20Gbps.

In the $5 \mathrm{G}$ era, the horizontal traffic between base stations will far exceed that of the LTE era. The delay requirement is even within $1 \mathrm{~ms}$, which is much lower than the ideal delay (10 ms) between LTE base stations defined by the 3GPP. LTE era, the third floor is generally high, mainly deployed in the convergence of key nodes or core layer. In the era of 5G, on the one hand, more base stations will be hung on the third floor and the number of routing entries will be larger. In the traffic scheduling task of Layer 3 equipment, if the three-layer high-rise equipment and the third-floor equipment will be overwhelmed, once the three-layer node fails, On the other hand, there is a large delay in the traffic flow of the three-storey high-level equipment. This will not meet the requirements of the traffic flow delay between the base stations in the $5 \mathrm{G}$ era. In the era of 5G, three domains of RAN control domain, bearer network control domain, and core network control domain cooperate to achieve end-to-end network control, network coordination and service deployment so as to implement any topology, any service, any service quality requirement, any forwarding requirement, any Failure of network protection switching, etc., and open to third parties to create an open network platform. In order to realize the coordination of three domains, SDN deployment of bearer network is imperative.

$5 G$ carries the architecture of forwarding and control separation. The forwarding plane is virtualized to assume the form of "one physical architecture and multiple networking architectures." By slicing the network and building different logical network instances as needed, the $5 \mathrm{G}$ needs to support both wired and wireless network resources Unified collaborative scheduling to achieve on-demand service based on the business chain.

The SDN realizes the separation of forwarding and control. The work of the control plane of the bearer equipment is completely transferred to the SDN controller, so as to realize the centralized control of the entire network equipment. Deploying SDN can bring the following benefits to the network:

1) The deployment of SDN controller greatly reduces the burden on the bearer equipment, and enables the bearer equipment to focus on the service forwarding without having to constantly run the control plane process which consumes a great amount of power and has high requirements on equipment functions. The energy consumption and function requirements of the equipment are greatly reduced.

2) Due to the deployment of the SDN controller, the calculation of Layer 3 routing is performed by the SDN controller. The label table is also generated by the SDN controller. The bearer device does not need to operate a complicated routing protocol process, and the requirements for bearer devices are greatly reduced. In this way, the three-layer capability in the $4 \mathrm{G}$ network is weak, and the equipment with a good forwarding capability can continue to play a role in the 5G era and protect the investment in equipment of operators.

3) Future network development is based on SDN / NFV. Through the network orchestration and management system, the network is sliced according to specific scene requirements, and the network functions are tailored and on-demand network deployment to meet the requirements of different scenarios such as bandwidth, delay and service quality Differentiation requirements. 
4) IEEE 1588V2 Deployment

$5 \mathrm{G}$ era, the requirements of the base station synchronization more stringent than the $4 \mathrm{G}$ era. Since the number of base stations in the $5 \mathrm{G}$ era is quite different from the number of base stations in the $4 \mathrm{G}$ era, the method of mainly realizing the frequency and time synchronization between base stations in the 3G / 4G era in the 3G / 4G era becomes extremely uneconomical in the 5G era and transmits frequency and time synchronization through the terrestrial transmission network Become inevitable, the deployment of IEEE 1588V2 is inevitable.

\section{Conclusions}

This article introduces the dramatic increase of capacity, connection and delay sensitivity caused by the change of architecture, air interface and terminal in the evolution of 4G to 5G. It analyzes the impact of ultra-high speed and large capacity mobile broadband access on the transport bearer network Higher bandwidth and larger capacity requirements, put forward new requirements for the transmission performance, interface technology and networking architecture of the pre-transmission and post-transmission networks. The article estimates the bandwidth of the IPRAN transmission network, analyzes the development and evolution of the ultra-large-capacity 100G interfaces, high-precision time synchronization, NGFI, IPRAN and other technologies, and focuses on the gradual Shen three-tier equipment, network architecture moving down the third floor, large-capacity and flexible network development and transport layer core layer, convergence layer, access layer evolution of the development and so on, in order to meet the 5G even more long-term Network development needs. The arrival of $5 G$ at the same time provides a favorable opportunity for the introduction of SDN. Therefore, the flattening of the bearer network, the convergence of multiple technologies such as packet and light, the convergence of fixed and mobile, and the fusion of the prequel and the backhaul are the key points that need to be considered afterwards in the 5G network.

\section{References}

[1] Huang Yi-wen. Protection Scheme of PTN Bearer Network [J]. Telephone Designing Technology 2010 (1): 53-57.

[2] Shen Jianfeng, Shou Xinling. Application of PTN in Packet Transfer of Mobile Transport Network [J]. Telecommunications Engineering Technology and Standardization, 2010,23 (2): 48-51.

[3] Zhang Chengliang, Jing Ruiquan. PTN technology trends and networking applications [J]. Post and Telecommunications Design Technology, 2010 (3): 1-4.

[4] Lei Qiuyan, Zhang Zhizhong, Cheng Fang, et al.C-RAN based 5G wireless access network architecture [J]. Telecommunications Science, 2015 (1): 106-115.

[5] Zhao Huiling, Feng Ming, Shi Fan.SDN - An Important Trend of Future Network Evolution [J]. Telecommunications Science, 2012,28 (11): 1-5.

[6] Huo Xiaoli, Jing Ruiquan. CPRI link bearer scheme for centralized deployment of BBU [J]. Telecommunications Science, 2015 (8): 161-165 (in Chinese).

[7] Wang Changting, Han Dongmei.C-RAN transmission solution of new ideas [J]. Communication World, 2015 (6): 8-10.

[8] You Xiaohu, Pan Zhiwen, Gao Xiqi et al. Development Trend and Several Key Technologies of 5G Mobile Communication [J]. Chinese Science, 2014,44 (5): 551-563.

[9] He Hongzhi. An interference suppression method in C-RAN wireless network [J]. Journal of Chongqing University of Posts and Telecommunications: Natural Science, 2015 (4): 493-498.

[10] Wang Xiaoyun, Huang Yuhong, Cui Chunfeng, et al.C-RAN: Evolution towards a Green Future Wireless Access Network [J]. China Communications, 2010 (3): 107-112. 
[11] Editorial Telecommunication Engineering Technology and Standardization. ITU releases 5G schedule [J]. Telecommunications Engineering Technology and Standardization, 2015 (7): 67.

[12] IMT-2020 Propulsion Team .5G Vision and Requirements [Z] .2014.

[13] IMT 2020 Propulsion Group .5G White Paper on Wireless Technology Architecture [Z] .2014.

[14] ITU, Document 5D / TEMP / 625-E.IMT Vision-Frame work and Overall Objectives of the Future Development of IMT for 2020 and Beyond [S] .2015.

[15] IMT-2020 Propulsion Group .5G Concept White Paper [Z] .2015. 